\title{
Markers of endothelial injury and subclinical inflammation in children and adolescents with primary hypertension
}

\author{
PIOTR SKRZYPCZYK', ANNA OZIMEK ${ }^{1}$, ANNA OFIARA ${ }^{2}$, MICHAE SZYSZKA2, \\ JAROSŁAW SOŁTYSKI I , ANNA STELMASZCZYK-EMMEL ${ }^{3}$ ELŻBIETA GÓRSKA ${ }^{3}$, \\ MAEGORZATA PAŃCZYK-TOMASZEWSKA ${ }^{l}$ \\ 'Department of Pediatrics and Nephrology, Medical University of Warsaw, Warsaw, Poland \\ ${ }^{2}$ Student Scientific Group at the Department of Pediatrics and Nephrology, Medical University of Warsaw, Warsaw, Poland \\ ${ }^{3}$ Department of Laboratory Diagnostics and Clinical Immunology of Developmental Age, Medical University of Warsaw, Warsaw, Poland
}

\begin{abstract}
Introduction: Adhesion molecules: E-selectin and intercellular adhesion molecule 1 (ICAM-1) are well-established markers of endothelial injury. The aim of the study was to assess the relation between E-selectin and ICAM-1 and clinical and biochemical parameters in children and adolescents with primary hypertension $(\mathrm{PH})$.

Material and methods: In 77 patients with PH (15.04 \pm 2.62 years, 50 boys, 27 girls) we evaluated serum E-selectin, ICAM-1, and selected clinical and biochemical parameters including inflammatory indicators and ambulatory blood pressure monitoring (ABPM).

Results: The E-selectin concentration was $55.63 \pm 26.49 \mathrm{ng} / \mathrm{ml}$ and the ICAM-1 concentration was $302.17 \pm 67.14 \mathrm{ng} / \mathrm{ml}$. E-selectin and ICAM-1 correlated $(p<0.05)$ with BMI Z-score $(r=0.24$, $r=0.29)$, ICAM-1 also with uric acid $(r=0.35), H D L$-cholesterol $(r=-0.28)$, platelet-to-lymphocyte ratio $(r=0.26)$, and systolic and mean blood pressure variability $(r=0.24, r=0.24)$; in boys ICAM-1 correlated with mean platelet volume $(r=0.29)$. In multivariate analysis the only significant predictor of E-selectin was mean arterial pressure during 24 hours $(\beta=0.329,95 \%$ CI: 0.012-0.646) and of ICAM-1 - uric acid ( $\beta=0.430$, 95\% CI: 0.040-0.819). In 27 children with newly diagnosed PH E-selectin correlated negatively with diastolic blood pressure dipping $(r=-0.54, p=0.004)$ and positively with ambulatory arterial stiffness index $(r=0.51, p=0.012)$.

Conclusions: Elevated mean arterial pressure and hyperuricemia are risk factors of endothelial damage in paediatric patients with primary hypertension. In children with untreated primary hypertension there may be a relation between endothelial damage and disturbed circadian blood pressure profile.
\end{abstract}

Key words: children, ICAM-1, adolescents, E-selectin, endothelial injury, primary hypertension, subclinical inflammation.

(Centr Eur J Immunol 2019; 44 (3): 253-261)

\section{Introduction}

Primary hypertension $(\mathrm{PH})$ is a dominant form of arterial hypertension in teenagers, and its incidence in children and adolescents is rising as a consequence of increasing prevalence of obesity and excessive salt intake $[1,2]$. PH in considered to be not only a disease of the cardiovascular system but also a systemic disorder involving inappropriate body composition and fatty tissue distribution, sympathetic overdrive, immune system activation, and premature (early) vascular aging with endothelial injury [3]. Endothelium forms an internal lining of all blood vessels and is an extremely important regulator of blood pressure and tissue perfusion. Arterial hypertension causes shear stress to endothelial cells leading to their activation and dysfunction [4].

Adhesion molecules include two families: the selectin family (E-selectin, P-selectin, and L-selectin) and the immunoglobulin family (intercellular and vascular adhesion molecules - ICAM-1 and VCAM-1) [5]. They are localised on the membranes of activated cells: leukocytes, platelets, and endothelium. Soluble forms of these adhesion molecules originate either from enzymatic cleavage or alternative splicing of the messenger RNA. E-selectin and

Correspondence: Piotr Skrzypczyk, MD, PhD, Department of Pediatrics and Nephrology, Medical University of Warsaw,

63A Żwirki i Wigury St., 02-091 Warsaw, Poland, e-mail: pskrzypczyk@wum.edu.pl

Submitted: 14.07.2019; Accepted: 21.07.2019 
ICAM-1 are adhesion molecules, produced almost exclusively by activated endothelial cells. E-selectin mediates leukocyte rolling on the endothelium and platelet-leukocyte interaction, whereas ICAM-1 is a molecule responsible for leukocyte migration into subendothelial layers of the vascular wall $[4,5]$. Soluble ICAM-1 concentration was found to be linked to future incidence of coronary heart disease [6], and E-selectin concentration was strongly related to traditional cardiovascular risk factors [7].

Subclinical inflammation and endothelial activation contribute to the development and progression of atherosclerosis [3]. Over the last decades, a vast number of studies have implicated endothelial dysfunction in the progression of atherosclerosis and target organ damage in primary hypertension $[3,6,7]$. Several biomarkers have been established to reflect the status of subclinical inflammation in cardiovascular diseases, including high-sensitivity C-reactive protein (CRP), interleukin (IL)-6, and complete blood count-derived indices [8].

There are only a few studies on markers of endothelial injury and subclinical inflammation in paediatric patients with PH $[9,10]$. In our previous study we showed a significant relation between blood pressure, urinary albumin loss, and subclinical inflammation in adolescents with PH [8].

Little is known about factors predisposing to endothelial damage in paediatric patients with $\mathrm{PH}$. Also, there is no data on dependence between grade of subclinical inflammation and endothelial damage in these subjects. Unmasking these factors and dependences could result in interventional studies aimed at improvement of endothelial function and in implementation of therapeutic measures focusing at endothelial health. Thus, the aim of this study was to establish the relation between endothelial damage measured as E-selectin and ICAM-1 concentrations, subclinical inflammation evaluated from complete blood count, and clinical and biochemical parameters in children and adolescents with PH, including ambulatory blood pressure monitoring (ABPM).

\section{Material and methods}

In this cross-sectional study we analysed the clinical data of 77 paediatric patients aged from 6.58 to 17.92 years, with PH treated at one tertiary paediatric nephrology centre. The enrolment into the study took place between April 2016 and April 2018. The inclusion criterion was: arterial hypertension diagnosed according to Polish up-to-date guidelines $[1,2]$. The exclusion criteria were: secondary forms of hypertension, heart defects, vascular pathology, and acute infections (temporary exclusion).

Venous peripheral blood was collected after overnight fasting, centrifuged to obtain serum, and stored at $-80^{\circ} \mathrm{C}$ until analysis. Concentration of markers of endothelium injury was measured in serum samples using enzyme-linked immunosorbent assays (ELISA) (sICAM-1/CD54 ELISA
Kit catalogue number: 950.540.096, sE-selectin/CD62E/ ELAM-1 ELISA Kit catalogue number: 850.530.096, both Diaclone SAS, Besancon Cedex, France; ng/ml). Complete blood count was performed using a Coulter LH 780 haematological analyser (Beckman Coulter Inc., Brea, CA, USA), and the following markers of subclinical inflammation were analysed: numbers of neutrophils

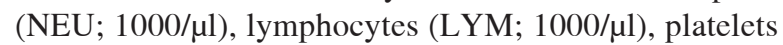
(PLT; 1000/ $\mu \mathrm{l})$, mean platelet volume (MPV; fl), and neutrophil-to-lymphocyte and platelet-to-lymphocyte ratios (NLR and PLR, respectively).

In addition, the following clinical parameters were assessed: age (years), sex, duration of PH (months), height $(\mathrm{cm})$, weight $(\mathrm{kg})$, and body mass index (BMI; $\left.\mathrm{kg} / \mathrm{m}^{2}\right)$. Anthropometric variables were expressed as $Z$-scores [11]. Overweight and obesity were defined in accordance with World Health Organisation definitions as BMI Z-score values $>1$ and $>2$, respectively.

Peripheral office blood pressure was measured using an oscillometric device (Welch Allyn VSM Patient Monitor 300, Welch Allyn Inc., Skaneateles Falls, NY, USA) and expressed as $\mathrm{mm} \mathrm{Hg}$ and $\mathrm{Z}$-score [12]. 24-hour ABPM was performed using a SUNTECH OSCAR 2 device (SunTech Medical, Inc., Morrisville, NC, USA) and interpreted according to American Heart Association (AHA) guidelines [13]. The methodology and data interpretation were described in detail previously [8]. In the final analysis we included the following ABPM-derived parameters: systolic, diastolic, and mean arterial pressure (SBP, DBP, MAP) during 24 hours (mm Hg), MAP 24 h Z-score [13], pulse pressure ( $\mathrm{mm} \mathrm{Hg}$ ), heart rate (beats per minute), SBP and DBPL load during 24 hours (\%), nocturnal blood pressure dip (\%), blood pressure variability (standard deviation of all measurements) (SBPV, DBPV), and ambulatory arterial stiffness index (AASI) [14]. Disturbed circadian blood pressure rhythm was defined as nocturnal SBP or DBP dip less than $10 \%$ [13].

The following biochemical parameters were evaluated: serum creatinine [mg/dl], uric acid [mg/dl], total low-density lipoprotein (LDL), and high-density lipoprotein (HDL) cholesterol (mg/dl), triglycerides (mg/dl), calcium (mg/ $\mathrm{dl})$, phosphorus (mg/dl), 25 hydroxyvitamin D (25[OH)] $\mathrm{D} ; \mathrm{ng} / \mathrm{ml}$ ), alkaline phosphatase activity (ALP; IU/l), and daily urinary albumin loss (albumin excretion rate - AER; $\mathrm{mg} / 24 \mathrm{~h}$ ). Biochemical parameters were assessed by dry chemistry (Ortho Clinical Diagnostics, Raritan, NJ, USA), and vitamin D by chemiluminescence (ARCHITECT i1000SR, Abbott Laboratories, Abbott Park, IL, USA). Glomerular filtration rate (GFR) was calculated according to the Schwartz formula [15]. Hyperuricemia was defined as uric acid $>6.0 \mathrm{mg} / \mathrm{dl}$, hypercholesterolemia as cholesterol $\geq 200 \mathrm{mg} / \mathrm{dl}$, and hypertriglyceridemia as triglyceride $\geq 100 \mathrm{mg} / \mathrm{dl}$ (children aged 0-9 years) or $\geq 130 \mathrm{mg} / \mathrm{dl}$ (children aged 10-19 years) [16]. Abnormal AER was defined as albuminuria $>30 \mathrm{mg} / 24 \mathrm{~h}[1,2]$. 
The research project was approved by the local Ethics Committee (approval No. KB/58/2016). All procedures were performed in accordance with the Declaration of Helsinki on the treatment of human subjects. Informed consent was obtained from all participants ( $\geq 16$ years) and their representatives.

Statistical elaboration was performed using Dell Statistica 13.0 PL software (Dell Inc., AlisoViejo, CA, USA). Variables were presented as the mean \pm standard deviation (SD) and interquartile range (IQR). Normality of data distribution was tested using Shapiro-Wilk test. The following tests were used (depending on data distribution): Student's $t$-test, $U$ Mann-Whitney test, ANOVA, Kruskal-Wallis test, Pearson linear correlation, Spearman rank correlation, and Fisher's exact test. Multivariate analysis was performed using a general regression model. Parameters that correlated with markers of endothelial injury with $p<0.300$ in univariate analysis were included in the final model. A $p$-value $<$ 0.05 was considered statistically significant.

\section{Results}

Clinical parameters in the studied children are presented in Table 1. Overweight was revealed in $31(40.3 \%)$ and obesity in $11(14.3 \%)$ patients. In the study group there were $27(35.1 \%)$ patients with newly diagnosed and untreated PH (patients referred for the first time to the Department) and 50 patients already treated for hypertension. Among the 50 patients treated for $\mathrm{PH} 33$ received antihypertensive medications and 17 patients were treated only with non-pharmacological measures. In the subgroup of subjects on antihypertensive drugs, 12 were treated with more than one antihypertensive drug (10 patients with two medications, and two patients with three medications). The results of ambulatory blood pressure monitoring are presented in Table 2. Fifteen among 27 (55.6\%) patients with newly diagnosed hypertension had isolated systolic hypertension. Ten among $33(30.3 \%)$ patients receiving pharmacological treatment and nine among 17 (52.9\%) on non-pharmacological treatment had poor control of hypertension in ABPM. Disturbed circadian blood pressure profile was revealed in $30(39.0 \%)$ patients. Markers of endothelial damage, markers of subclinical inflammation, and remaining biochemical results are depicted in Table 3. Hyperuricemia was found in 29 (37.7\%), hypercholesterolemia in nine $(11.7 \%)$, and hypertriglyceridemia in $16(20.8 \%)$ patients. Increased albumin urinary excretion was revealed in 10 (13.0\%) children with $\mathrm{PH}$.

Concentrations of markers of endothelial injury in different groups of patients are presented in Figures 1 and 2. The subgroups of untreated patients, patients treated with antihypertensive medications, and patients treated only with non-pharmacological measures did not differ significantly in terms of E-selectin and ICAM-1 (55.13 \pm 23.29 vs. 53.17 \pm 25.44 vs. $61.18 \pm 33.33[\mathrm{ng} / \mathrm{ml}], p=0.600$, and 304.29
Table 1. Clinical data of children with primary hypertension

\begin{tabular}{|c|c|}
\hline Parameter & Value \\
\hline Age, years & $15.04 \pm 2.62(13.83-17.08)$ \\
\hline Sex (boys/girls), $n(\%)$ & $50 / 27(64.9 / 35.1)$ \\
\hline $\begin{array}{l}\text { Duration of arterial hypertension, } \\
\text { months }\end{array}$ & $17.63 \pm 19.09(5-26)$ \\
\hline BMI, $\mathrm{kg} / \mathrm{m}^{2}$ & $24.99 \pm 5.14(21.64-27.47)$ \\
\hline BMI Z-score & $0.99 \pm 0.99(0.44-1.62)$ \\
\hline $\mathrm{SBP}, \mathrm{mm} \mathrm{Hg}$ & $134.21 \pm 13.32(124-145)$ \\
\hline SBP Z-score & $1.80 \pm 1.17(0.85-2.60)$ \\
\hline DBP, $\mathrm{mm} \mathrm{Hg}$ & $80.53 \pm 10.26(74-87)$ \\
\hline DBP Z-score & $1.18 \pm 0.94(0.52-1.90)$ \\
\hline Antihypertensive medications, $n(\%)$ & $33(42.9)$ \\
\hline Calcium channel antagonists & 24 \\
\hline $\begin{array}{l}\text { Angiotensin converting enzyme } \\
\text { inhibitors }\end{array}$ & 15 \\
\hline AT1 receptor antagonists & 3 \\
\hline$\beta$-adrenolytics & 3 \\
\hline Diuretics & 1 \\
\hline$\alpha$-adrenolytics & 1 \\
\hline
\end{tabular}

$\overline{S B P}$ - systolic blood pressure, DBP - diastolic blood pressure, BMI - body mass index, ATI - angiotensin II receptor type 1

Table 2. Results of ambulatory blood pressure monitoring in children with primary hypertension

\begin{tabular}{lr}
\hline 24-hour ambulatory blood pressure monitoring \\
\hline Systolic blood pressure during $24 \mathrm{~h}, \mathrm{~mm} \mathrm{Hg}$ & $128.17 \pm 11.03(118-136)$ \\
\hline Diastolic blood pressure during $24 \mathrm{~h}, \mathrm{~mm} \mathrm{Hg}$ & $70.84 \pm 7.43(65-76)$ \\
\hline Mean arterial pressure during 24 h, mm Hg & $89.95 \pm 8.03(85-96)$ \\
\hline Mean arterial pressure during 24 h Z-score & $1.12 \pm 1.44(0.03-1.84)$ \\
\hline Pulse pressure during 24 h, mm Hg & $57.53 \pm 8.03(52-63)$ \\
\hline Heart rate during 24 h, beats per minute & $77.00 \pm 13.41(71-87)$ \\
\hline Systolic blood pressure load during 24 h, \% & $39.26 \pm 27.06(16-61)$ \\
\hline Diastolic blood pressure load during 24 h, \% & $23.37 \pm 21.06(7-32)$ \\
\hline Systolic blood pressure variability & $13.14 \pm 2.73(11.2-14.6)$ \\
\hline Diastolic blood pressure variability & $11.16 \pm 3.10(9.5-12.4)$ \\
\hline Mean blood pressure variability & $11.07 \pm 2.78(9.5-12.0)$ \\
\hline Night-time systolic blood pressure dip, \% & $10.84 \pm 5.72(7.5-14.8)$ \\
\hline Night-time diastolic blood pressure dip, \% & $15.35 \pm 8.23(11.0-21.0)$ \\
\hline Ambulatory arterial stiffness index (AASI) & $0.38 \pm 0.12(0.30-0.45)$ \\
\hline
\end{tabular}

\pm 53.13 vs. $305.67 \pm 77.92$ vs. $292.02 \pm 67.39[\mathrm{ng} / \mathrm{ml}]$, $p=0.782$, respectively). Also, no relation between treatment with the two most commonly used antihypertensive drugs (calcium channel blockers and angiotensin converting enzyme inhibitors) and the concentration of markers of endothelial damage was found. E-selectin was significantly 
Table 3. Markers of endothelial injury, markers of subclinical inflammation, and biochemical data in studied children

\begin{tabular}{|c|c|}
\hline Parameter & Value \\
\hline E-selectin, $\mathrm{ng} / \mathrm{ml}$ & $55.63 \pm 26.49(36.22-69.57)$ \\
\hline ICAM-1, ng/ml & $302.17 \pm 67.14(259.50-328.00)$ \\
\hline 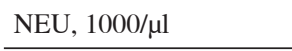 & $3.81 \pm 1.70(2.60-4.60)$ \\
\hline 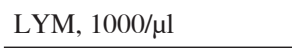 & $2.27 \pm 0.78(1.78-2.60)$ \\
\hline PLT, $1000 / \mu 1$ & $256.05 \pm 55.22(221-285)$ \\
\hline MPV, fl & $9.67 \pm 1.53(8.30-10.80)$ \\
\hline NLR & $1.84 \pm 1.05(1.16-2.15)$ \\
\hline PLR & $121.82 \pm 38.93(98.18-143.71)$ \\
\hline $\begin{array}{l}\text { GFR ac. to Schwartz, } \\
\mathrm{ml} / \mathrm{min} / 1.73 \mathrm{~m}^{2}\end{array}$ & $97.62 \pm 18.46(84.32-108.36)$ \\
\hline Uric acid, mg/dl & $5.99 \pm 2.01(4.8-6.9)$ \\
\hline Total cholesterol, mg/dl & $159.79 \pm 39.29(128-177)$ \\
\hline HDL cholesterol, mg/dl & $49.60 \pm 11.36(43.0-56.0)$ \\
\hline LDL cholesterol, mg/dl & $90.14 \pm 35.92(66.0-104.0)$ \\
\hline Triglycerides, mg/dl & $101.86 \pm 48.23(68.0-122.0)$ \\
\hline Albuminuria, mg/24 h & $27.37 \pm 46.10(5.28-21.20)$ \\
\hline
\end{tabular}

higher in 50 boys than in 27 girls ( $60.02 \pm 26.56$ vs. 47.49 \pm 24.80 [ng/ml], $p=0.047$ ), but there was no significant difference in ICAM-1 between boys and girls (312.63 \pm 72.44 vs. $282.80 \pm 51.86[\mathrm{ng} / \mathrm{ml}], p=0.062$ ).

The relation between markers of endothelial injury and selected clinical, biochemical, and inflammatory parameters are depicted in Table 4. In the whole group of 77 patients E-selectin and ICAM-1 concentrations correlated with BMI $Z$-score. ICAM-1 correlated also with uric acid, HDL-cholesterol, ALP, PLR, and with SBPV

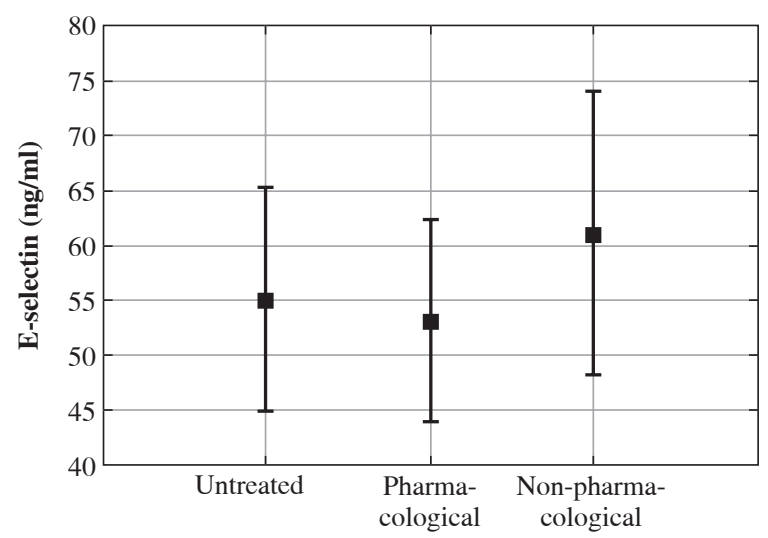

Fig. 1. E-selectin in children with primary hypertension $(p=0.600)$ and MAPV; additionally, in boys ICAM-1 correlated with MPV ( $r=0.29, p=0.038)$. The results of multivariate analysis are shown in Table 5. In multivariate analysis using a general regression model the only significant predictors of markers of endothelial injury were mean arterial pressure during 24 hours in ABPM expressed as Z-score for E-selectin ( $\beta=0.329,95 \%$ CI: 0.012-0.646, $p=0.042$ ) and uric acid for ICAM- 1 ( $\beta=0.430,95 \%$ CI: 0.040 $0.819, p=0.031)$.

In the subgroup of 27 patients with newly diagnosed hypertension there were no differences in concentrations of E-selectin and ICAM-1 between 15 children with isolated systolic hypertension and 12 children with systolic and diastolic hypertension. In this subgroup of 27 patients E-selectin correlated negatively with DBP dipping $(r=-0.54$, $p=0.004)$ and positively with AASI $(r=0.51, p=0.012)$.

\section{Discussion}

Our cross-sectional analysis revealed that the strongest predictors of endothelial damage in pediatric patients with primary hypertension were MAP in ABPM (for E-selectin) and serum uric acid (for ICAM-1). Additionally, overweight and obesity, low HDL-cholesterol, increased BP variability, and higher degree of subclinical inflammation may also be factors predisposing to endothelial dysfunction in this group of patients. Interestingly, in the subgroup of untreated, newly diagnosed patients we found also positive correlations of E-selectin with night-time DBP dipping, and most importantly with stiffness evaluated as AASI.

The Stanislas study evaluated the concentration of adhesion molecules in healthy children and adults [17]. Both ICAM-1 and E-selectin were inversely related to age in paediatric patients, but this relationship disappeared in multivariate analysis for E-selectin [17]. There was no relation between sex and level of adhesion molecules. In our cohort we found no relation of these biomarkers with age, and the relationship with sex was not confirmed in multi-

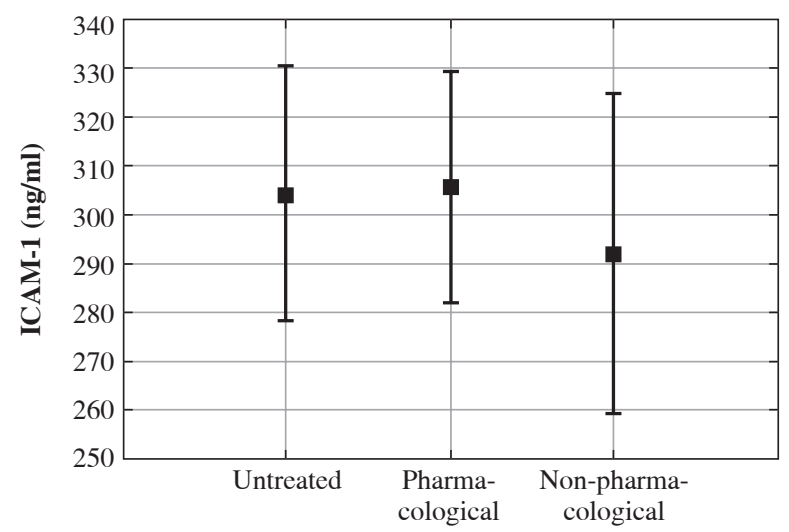

Fig. 2. Intercellular adhesion molecule (ICAM-1) in children with primary hypertension $(p=0.782)$ 
Table 4. The correlation between markers of endothelial injury and selected clinical, biochemical, and inflammatory indicators in children with primary hypertension

\begin{tabular}{|c|c|c|c|c|}
\hline \multirow[t]{3}{*}{ Indicators } & \multicolumn{4}{|c|}{ Markers of endothelial injury } \\
\hline & \multicolumn{2}{|c|}{ ICAM-1 (ng/ml) } & \multicolumn{2}{|c|}{ E-selectin (ng/ml) } \\
\hline & $r$ & $p$ & $r$ & $p$ \\
\hline ICAM-1, ng/ml & - & - & 0.33 & 0.003 \\
\hline E-selectin, ng/ml & 0.33 & 0.003 & - & - \\
\hline Age, years & 0.16 & 0.172 & -0.18 & 0.110 \\
\hline Duration of hypertension, months & 0.01 & 0.993 & 0.03 & 0.821 \\
\hline BMI Z-score & 0.29 & 0.011 & 0.24 & 0.037 \\
\hline Office SBP Z-score & 0.08 & 0.504 & -0.02 & 0.838 \\
\hline Office DBP Z-score & 0.06 & 0.626 & -0.06 & 0.581 \\
\hline GFR, $\mathrm{ml} / \mathrm{min} / 1.73 \mathrm{~m}^{2}$ & 0.12 & 0.287 & -0.15 & 0.204 \\
\hline Uric acid, mg/dl & 0.35 & 0.003 & 0.17 & 0.148 \\
\hline Total cholesterol, mg/dl & 0.05 & 0.665 & 0.14 & 0.245 \\
\hline HDL cholesterol, mg/dl & -0.28 & 0.020 & -0.09 & 0.475 \\
\hline LDL cholesterol, mg/dl & 0.19 & 0.113 & 0.19 & 0.113 \\
\hline Triglycerides, mg/dl & 0.07 & 0.549 & 0.17 & 0.163 \\
\hline Vitamin D, ng/ml & -0.12 & 0.316 & 0.09 & 0.460 \\
\hline Alkaline phosphatase, IU/l & 0.39 & $<0.001$ & 0.20 & 0.086 \\
\hline Albuminuria, mg/24 h & -0.08 & 0.535 & 0.05 & 0.741 \\
\hline NEU, $1000 / \mu \mathrm{l}$ & 0.06 & 0.616 & 0.06 & 0.616 \\
\hline $\mathrm{LYM}, 1000 / \mu \mathrm{l}$ & -0.10 & 0.389 & -0.06 & 0.607 \\
\hline PLT, $1000 / \mu 1$ & 0.17 & 0.132 & -0.03 & 0.818 \\
\hline MPV (fl) & 0.08 & 0.490 & -0.01 & 0.969 \\
\hline NLR & 0.13 & 0.265 & 0.09 & 0.460 \\
\hline PLR & 0.26 & 0.025 & 0.06 & 0.602 \\
\hline MAP 24-h Z-score & 0.06 & 0.620 & 0.18 & 0.122 \\
\hline SBP load (\%) & 0.05 & 0.649 & 0.11 & 0.323 \\
\hline DBP load (\%) & 0.08 & 0.492 & 0.11 & 0.339 \\
\hline SBP variability & 0.24 & 0.037 & 0.03 & 0.825 \\
\hline DBP variability & 0.22 & 0.058 & -0.01 & 0.907 \\
\hline MAP variability & 0.24 & 0.038 & 0.06 & 0.586 \\
\hline Night-time SBP dip (\%) & 0.11 & 0.328 & 0.12 & 0.314 \\
\hline Night-time DBP dip (\%) & 0.09 & 0.429 & 0.01 & 0.969 \\
\hline AASI & -0.05 & 0.672 & 0.15 & 0.230 \\
\hline
\end{tabular}

ICAM - intercellular adhesion molecule, BMI - body mass index, SBP - systolic blood pressure, DBP - diastolic blood pressure, GFR - glomerular filtration rate, $H D L$ - high-density lipoprotein, LDL - low-density lipoprotein, NEU - neutrophils, LYM - lymphocytes, PLT - platelets, MPV - mean platelet volume, NLR neutrophil-to-lymphocyte ratio, PLR - platelet-to-lymphocyte ratio, AASI-ambulatory arterial stiffness index

variate analysis. The discrepancy between our results and results in healthy individuals must be analysed with caution. It is probable that other factors determine endothelial function in hypertensive and normotensive individuals.

There is a pathophysiological link between inflammation and concentration of circulating adhesion molecules.
Leukocytes secrete cytokines that stimulate expression of adhesion molecules on the membrane of endothelial cells, thus facilitating adhesion and transfer of leukocytes into the arterial wall. Such a link was already proven in adults for TNF- $\alpha$ and E-selectin [17] and for CRP and ICAM-1 [18]. Also, in obese paediatric patients there was a positive 
Table 5. Multivariate analysis of factors influencing concentrations of E-selectin and ICAM-1 in children with primary hypertension

\begin{tabular}{|c|c|c|c|}
\hline Analysed parameter & $\beta$ & $95 \%$ confidence interval & $p$ \\
\hline \multicolumn{4}{|c|}{ E-selectin } \\
\hline MAP 24-h Z-score & 0.329 & $(0.012-0.646)$ & $0.042^{*}$ \\
\hline Alkaline phosphatase, IU/l & 0.351 & $(-0.111-0.814)$ & 0.132 \\
\hline BMI Z-score & 0.197 & $(-0.157-0.552)$ & 0.268 \\
\hline GFR, $\mathrm{ml} / \mathrm{min} / 1.73 \mathrm{~m}^{2}$ & -0.187 & $(-0.627-0.253)$ & 0.395 \\
\hline AASI & 0.134 & $(-0.191-0.459)$ & 0.409 \\
\hline Triglyceride, $\mathrm{mg} / \mathrm{dl}$ & 0.120 & $(-0.276-0.515)$ & 0.545 \\
\hline Age, years & -0.143 & $(-0.628-0.342)$ & 0.555 \\
\hline Uric acid, mg/dl & -0.106 & $(-0.578-0.366)$ & 0.654 \\
\hline Sex & 0.045 & $(-0.377-0.466)$ & 0.831 \\
\hline Cholesterol, mg/dl & 0.139 & $(-1.315-1.593)$ & 0.848 \\
\hline LDL cholesterol, $\mathrm{mg} / \mathrm{dl}$ & -0.027 & $(-1.484-1.429)$ & 0.970 \\
\hline \multicolumn{4}{|c|}{ ICAM-1 } \\
\hline Uric acid, mg/dl & 0.430 & $(0.040-0.819)$ & $0.0313^{*}$ \\
\hline GFR, $\mathrm{ml} / \mathrm{min} / 1.73 \mathrm{~m}^{2}$ & 0.361 & $(-0.052-0.773)$ & 0.085 \\
\hline PLR & 0.164 & $(-0.132-0.461)$ & 0.270 \\
\hline DBP variability & -0.548 & $(-1.932-0.836)$ & 0.430 \\
\hline Sex & 0.140 & $(-0.222-0.502)$ & 0.440 \\
\hline MAP variability & 0.586 & $(-1.318-2.490)$ & 0.538 \\
\hline BMI Z-score & 0.077 & $(-0.251-0.405)$ & 0.638 \\
\hline SBP variability & 0.180 & $(-0.636-0.995)$ & 0.659 \\
\hline Age, years & 0.065 & $(-0.365-0.496)$ & 0.761 \\
\hline NLR & 0.038 & $(-0.252-0.327)$ & 0.793 \\
\hline Platelets, $1000 / \mu \mathrm{l}$ & 0.029 & $(-0.311-0.369)$ & 0.865 \\
\hline LDL cholesterol, $\mathrm{mg} / \mathrm{dl}$ & -0.024 & $(-0.334-0.285)$ & 0.875 \\
\hline HDL cholesterol, mg/dl & -0.017 & $(-0.323-0.290)$ & 0.913 \\
\hline Alkaline phosphatase, IU/l & 0.014 & $(-0.357-0.385)$ & 0.941 \\
\hline
\end{tabular}

MAP - mean arterial pressure, BMI - body mass index, GFR - glomerular filtration rate, AASI-ambulatory arterial stiffness index, LDL - low-density lipoprotein, PLR - platelet-to-lymphocyte ratio, DBP - diastolic blood pressure, SBP - systolic blood pressure, NLR - neutrophil-to-lymphocyte ratio, HDL - high-density lipoprotein

relation between SBP, CRP, and VCAM-1 and between diastolic blood pressure, CRP, and ICAM-1 [10]. NLR and PLR, as well as MPV, are easily accessible indicators of subclinical inflammation. In our cohort, ICAM-1 correlated positively with PLR and, in boys, with MPV. In our previous study we found a positive relation between these indices and ambulatory blood pressure, albuminuria, and plasma renin activity in untreated adolescents with primary hypertension [8].

E-selectin and ICAM-1 correlated positively with BMI $Z$-score, ICAM-1 positively with uric acid, and negatively with HDL-cholesterol. The positive association between markers of endothelial injury and BMI is consistent with earlier observations in adults (E-selectin) $[7,17]$ and in children (E-selectin and ICAM-1) [9]. Increased levels of E-selectin in overweight, obesity, and dyslipidaemia could be explained by the adipocyte producing pro-inflammatory cytokines that induced E-selectin gene expression [19]. However, the relation between endothelial dysfunction and dyslipidaemia may be more complex because HDL-cholesterol was found to inhibit TNF- $\alpha$ and IL-1, cytokines that induce expression of adhesion molecules [20]. Accordingly, in our cohort there was a negative correlation of ICAM-1 and HDL-cholesterol. Similarly, in a study from Malaysia patients with low HDL-cholesterol were characterised by higher E-selectin and ICAM-1 concentrations [20]. Whereas, in the ELSA-Brasil study the highest E-selectin concentrations were associated with the highest total 
and LDL-cholesterol and triglycerides without differences in HDL-cholesterol [7]. In a paediatric study by Glowinska et al. total cholesterol and triglycerides correlated positively with E-selectin and ICAM-1 [9].

The increase in serum uric acid is considered to be not only a hallmark but also one of the triggering factors of PH [21]. Elevated serum uric acid may directly damage endothelial cells [22]. In our cohort there was a positive correlation between serum uric acid and ICAM-1, also confirmed by multivariate analysis. The same relation was found in obese prepubertal children [23]. Similarly, a negative relation between flow-mediated dilation and serum uric acid was found in obese boys [24]. There remains an unanswered question, whether lowering uric acid can reverse the negative phenomena observed in $\mathrm{PH}$, including endothelial injury. Feig showed that treatment with allopurinol can normalise blood pressure in hypertensive adolescents [25], but a recently published trial failed to prove a beneficial role of allopurinol in normalisation of endothelial dysfunction in adults with PH [26].

Endothelial dysfunction promotes vascular smooth muscle proliferation and fibrosis of arterial wall, thus leading to impaired arterial contractility. The positive correlation found in our untreated hypertensive subjects between E-selectin and AASI might confirm this phenomenon. AASI is an ABPM-derived marker of arterial elasticity [14]. Although its clinical value in children remains unclear, it was found to be elevated in children with hypertension [27] and IgA nephropathy [28]. Nevertheless, the small number of patients (only 27 untreated children), the relationship found only in univariate analysis, and the uncertain significance of AASI preclude drawing final conclusions on the role of E-selectin as a serum marker of arterial stiffness. Eikendal et al. found a similar relation between E-selectin and aortal pulse wave velocity (PWV) in young adults [29]. In contrast, in a study by de Faria P-selectin and VCAM-1 but not E-selectin and ICAM-1 were predictors of increased arterial stiffness assessed by applanation tonometry [30].

In our patients, multivariate analysis unmasked a relationship between E-selectin and mean arterial pressure in ABPM. Positive correlations between ICAM-1, E-selectin, and SBP and DBP in ABPM were revealed in paediatric patients with type 1 diabetes mellitus [9, 31]. Also, E-selectin correlated positively with office blood pressure in adult individuals [7]. In addition, an association was found between genetic variants of E-selectin and risk of $\mathrm{PH}$ [32]. This positive correlation between blood pressure and markers of endothelial activation and injury highlights phenomenon of negative impact of blood pressure ("shear stress") on endothelium. Of note, in our group a relationship between endothelial dysfunction was found only for MAP during 24 hours. Oscillometric devices directly measure the mean arterial pressure, and MAP during 24 hours seems to reflect most precisely the cardiovascular burden as shown in the ESCAPE study [33].
Our patients showed a positive relation between ICAM-1 and blood pressure variability. Increased blood pressure variability is an independent cardiovascular risk factor [34]. In adults a positive correlation between blood pressure variability and E-selectin [35] and ICAM-1 [36] has already been found. To the best of our knowledge, this is the first study to confirm this relation in paediatric patients with $\mathrm{PH}$. The hypothesis that short-term blood pressure oscillations lead to vascular damage may be a causative link between these two phenomena [35]. In 27 individuals with newly diagnosed hypertension we found a negative correlation between E-selectin and nighttime diastolic blood pressure dip. Paediatric patients with PH and disturbed circadian blood pressure profile are at risk of left ventricular hypertrophy [37]. Moreover, many studies showed that nocturnal hypertension is a risk factor for kidney function decline [38]. There is still debate whether endothelial dysfunction leads to blunted night-time dip by increasing arterial stiffness. Conversely, according to opposite theory, increased night-time shear stress damages endothelium. The cross-sectional character of our study precludes drawing final conclusions on this topic.

Increased urinary albumin loss is not only a marker of glomerular hyperpermeability but also reflects endothelial dysfunction with strong correlations between albuminuria and cardiovascular risk [39]. In diabetic adults ICAM-1 showed a positive correlation with AER. Moreover, individuals with the highest values of ICAM-1 had the highest increase in AER after three years [40]. No relation was revealed between markers of endothelial injury and albuminuria in our cohort. Of note, there are no data linking albuminuria in children with hard cardiovascular end-points in prospective observation, and the American Academy of Pediatrics resigned from routine assessment of albuminuria in hypertensive individuals [41].

In our cohort ALP activity was positively associated with E-selectin and ICAM-1 concentrations. The same correlation was observed also in healthy individuals [17] and for ICAM-1 in patients with hepatic disorders [42]. It must be emphasised that ALP activity remained within normal limits in all our subjects, and none of them suffered from bone or hepatic pathology. We did not find in the literature any explanation for the positive relation between endothelial adhesion molecules and ALP in a physiological state.

The limitations of our research are a lack of analysis of adhesion molecules in healthy individuals, indirect analysis of arterial stiffness only by ABPM, and assessment of target-organ damage limited to albumin excretion. In addition, this was a cross-sectional study, therefore a causal relationship cannot be inferred. Also, the study group was not large and not uniform, especially in terms of the treatment used; thus, detailed analysis in subgroups was not possible and final firm conclusions cannot be drawn uncritically. Nevertheless, owing to the fact that we analysed a relatively large group of individuals and blood 
pressure is a continuous variable with arbitrary definition of hypertension, our result can be cautiously extrapolated to the whole paediatric population.

\section{Conclusions}

Elevated mean arterial pressure and hyperuricemia are risk factors of endothelial damage in paediatric patients with primary hypertension.

In children with untreated primary hypertension there may be a relation between endothelial damage and disturbed circadian blood pressure profile.

\section{The authors declare no conflict of interest.}

\section{References}

1. Litwin M, Niemirska A, Obrycki Ł, et al. (2018): Guidelines of the Pediatric Section of the Polish Society of Hypertension on diagnosis and treatment of arterial hypertension in children and adolescents. Arterial Hypertens 22: 45-73.

2. Żurowska A, Zwolińska D, Roszkowska-Blaim M, et al. (2015): Rekomendacje Polskiego Towarzystwa Nefrologii Dziecięcej (PTNFD) dotyczące postępowania z dzieckiem z podwyższonym ciśnieniem tętniczym. Forum Med Rodz 9: 349-375.

3. Litwin M, Feber J, Niemirska A, Michałkiewicz J (2016): Primary hypertension is a disease of premature vascular aging associated with neuro-immuno-metabolic abnormalities. Pediatr Nephrol 3: 185-194.

4. Endemann DH, Schiffrin EL (2004): Endothelial dysfunction. J Am Soc Nephrol 15: 1983-1992.

5. Derosa G, Maffioli P (2016): A review about biomarkers for the investigation of vascular function and impairment in diabetes mellitus. Vasc Health Risk Manag 12: 415-419.

6. Schmidt C, Hulthe J, Fagerberg B (2009): Baseline ICAM-1 and VCAM-1 are increased in initially healthy middle-aged men who develop cardiovascular disease during 6.6 years of follow-up. Angiology 60: 108-114.

7. de Almeida-Pititto B, Ribeiro-Filho FF, Bittencourt MS, et al. (2016): Usefulness of circulating E-selectin to early detection of the atherosclerotic process in the Brazilian Longitudinal Study of Adult Health (ELSA-Brasil). Diabetol Metab Syndr 8: 19.

8. Skrzypczyk P, Przychodzień J, Bombińska M, et al. (2018): Complete blood count-derived inflammatory markers in adolescents with primary arterial hypertension: a preliminary report. Centr Eur J Immunol 43: 434-441.

9. Glowinska B, Urban M, Peczynska J, Florys B (2005): Soluble adhesion molecules (sICAM-1, sVCAM-1) and selectins ( $\mathrm{sE}$ selectin, sP selectin, sL selectin) levels in children and adolescents with obesity, hypertension, and diabetes. Metabolism 54: 1020-1026.

10. Garanty-Bogacka B, Syrenicz M, Syrenicz A, et al. (2005): Serum markers of inflammation and endothelial activation in children with obesity-related hypertension. Neuro Endocrinol Lett 26: 242-246.

11. Kułaga Z, Litwin M, Tkaczyk M, et al. (2011): Polish 2010 growth references for school-aged children and adolescents. Eur J Pediatr 170: 599-609.
12. Kułaga Z, Litwin M, Grajda A, et al. (2012): Oscillometric blood pressure percentiles for Polish normal-weight schoolaged children and adolescents. J Hypertens 30: 1942-1954.

13. Flynn JT, Daniels SR, Hayman LL, et al. (2014): Update: ambulatory blood pressure monitoring in children and adolescents: a scientific statement from the American Heart Association. Hypertension 63: 1116-1135.

14. Li Y, Wang JG, Dolan E, et al. (2006): Ambulatory arterial stiffness index derived from 24-hour ambulatory blood pressure monitoring. Hypertension 47: 359-364.

15. Schwartz GJ, Muńoz A, Schneider MF, et al. (2009): New equations to estimate GFR in children with CKD. J Am Soc Nephrol 20: 629-637.

16. Expert panel on integrated guidelines for cardiovascular health and risk reduction in children and adolescents; National Heart, Lung and Blood Institute (2011): Expert panel on integrated guidelines for cardiovascular health and risk reduction in children and adolescents: summary report. Pediatrics 128 (Suppl 5): 213-256.

17. Ponthieux A, Herbeth B, Droesch S, et al. (2004): Biological determinants of serum ICAM-1, E-selectin, P-selectin and L-selectin levels in healthy subjects: the Stanislas study. Atherosclerosis 172: 299-308

18. Pasceri V, Willerson JT, Yeh ET (2000): Direct proinflammatory effect of C-reactive protein on human endothelial cells. Circulation 102: 2165-2168.

19. Ziccardi P, Nappo F, Giugliano G, et al. (2002): Reduction of inflammatory cytokine concentrations and improvement of endothelial functions in obese women after weight loss over one year. Circulation 105: 804-809.

20. Wan Ahmad WN, Sakri F, Mokhsin A, et al. (2015): Low serum high density lipoprotein cholesterol concentration is an independent predictor for enhanced inflammation and endothelial activation. PLoS One 10: e0116867.

21. Wang Y, Hu JW, Lv YB, et al. (2017): The role of uric acid in hypertension of adolescents, prehypertension and salt sensitivity of blood pressure. Med Sci Monit 23: 790-795.

22. Zoccali C, Maio R, Mallamaci F, et al. (2006): Uric acid and endothelial dysfunction in essential hypertension. J Am Soc Nephrol 17: 1466-1471.

23. Valle M, Martos R, Cańete MD, et al. (2015): Association of serum uric acid levels to inflammation biomarkers and endothelial dysfunction in obese prepubertal children. Pediatr Diabetes 16: 441-447.

24. Ishiro M, Takaya R, Mori Y, et al. (2013): Association of uric acid with obesity and endothelial dysfunction in children and early adolescents. Ann Nutr Metab 62: 169-176.

25. Feig DI, Soletsky B, Johnson RJ (2008): Effect of allopurinol on blood pressure of adolescents with newly diagnosed essential hypertension: A randomized trial. JAMA 300: 924-932.

26. Borgi L, McMullan C, Wohlhueter A, et al. (2017): Effect of uric acid-lowering agents on endothelial function: a randomized, double-blind, placebo-controlled trial. Hypertension 69: 243-248.

27. Simonetti GD, von Vigier RO, Wühl E, et al. (2008): Ambulatory arterial stiffness index is increased in hypertensive childhood disease. Pediatr Res 64: 303-307.

28. Skrzypczyk P, Mizerska-Wasiak M, Jerszow B, et al. (2017): Ambulatory arterial stiffness index, blood pressure variability, and nocturnal blood pressure dip in children with $\operatorname{IgA}$ and Henoch-Schönlein nephropathy. Clin Nephrol 87: 301-309.

29. Eikendal ALM, Bots ML, Gohar A, et al. (2018): Circulating levels of P-selectin and E-selectin relate to cardiovascular 
magnetic resonance-derived aortic characteristics in young adults from the general population, a cross-sectional study. J Cardiovasc Magn Reson 20: 54.

30. de Faria AP, Ritter AM, Sabbatini AR, et al. (2016): Deregulation of soluble adhesion molecules in resistant hypertension and its role in cardiovascular remodeling. Circ J 80: 11961201.

31. Machnica L, Deja G, Polanska J, et al. (2014): Blood pressure disturbances and endothelial dysfunction markers in children and adolescents with type 1 diabetes. Atherosclerosis 237: 129-134.

32. Wang Z, Xu Y, Chen S, et al. (2012): A common missense single nucleotide polymorphism in the E-selectin gene is significantly associated with essential hypertension in the Han population but only weakly associated in the Uygur population. Hypertens Res 35: 413-417.

33. Gimpel C, Wühl E, Arbeiter K, et al. (2009): ESCAPE Trial Group. Superior consistency of ambulatory blood pressure monitoring in children: implications for clinical trials. J Hypertens 27: 1568-1574.

34. Stevens SL, Wood S, Koshiaris C, et al. (2016): Blood pressure variability and cardiovascular disease: systematic review and metaanalysis. BMJ 354: i4098.

35. Tatasciore A, Zimarino M, Renda G, et al. (2008): Awake blood pressure variability, inflammatory markers and target organ damage in newly diagnosed hypertension. Hypertens Res 31: 2137-2146.

36. Ciobanu DM, Mircea PA, Bala C, Rusu A, et al. (2019): Intercellular adhesion molecule-1 (ICAM-1) associates with 24hour ambulatory blood pressure variability in type 2 diabetes and controls. Cytokine 116: 134-138.

37. Belsha CW, Wells TG, McNiece KL, et al. (1998): Influence of diurnal blood pressure variations on target organ abnormalities in adolescents with mild essential hypertension. Am J Hypertens 11: 410-417.

38. Tsioufis C, Andrikou I, Thomopoulos C, et al. (2011): Comparative prognostic role of nighttime blood pressure and nondipping profile on renal outcomes. Am J Nephrol 33: 277 288.

39. Stephen R, Jolly SE, Nally JV Jr, Navaneethan SD (2014): Albuminuria: when urine predicts kidney and cardiovascular disease. Cleve Clin J Med 81: 41-50.

40. Lin J, Glynn RJ, Rifai N, et al. (2008): Inflammation and progressive nephropathy in type 1 diabetes in the diabetes control and complications trial. Diabetes 31: 2338-2343.

41. Flynn JT, Kaelber DC, Baker-Smith CM, et al. (2017): Clinical practice guideline for screening and management of high blood pressure in children and adolescents. Pediatrics 140: e20171904.

42. Polzien F, Ramadori G (1996): Increased intercellular adhesion molecule-1 serum concentration in cholestasis. J Hepatol 25: 877-886. 